Gynäkologische Endokrinologie 2011 .

9:257-258

DOI 10.1007/s10304-011-0449-3

Online publiziert: 26. Oktober 2011

(c) Springer-Verlag 2011

Deutsche Menopause Gesellschaft e.V.

\section{Redaktion}

Deutsche Menopausegesellschaft e.V.

A.O. Mueck, Tübingen

P. Stute, Bern

P. Stute

Inselspital Bern

\title{
Anti-Müller-Hormon für die Prädiktion der Menopause
}

und FSH) bezüglich der Vorhersagekraft der Zeitspanne bis zur Menopause untersucht. Zum Zeitpunkt V2 waren 48 Frauen (19\%) postmenopausal. Die Autoren erstellten für die Beziehung zwischen altersspezifischem AMH-Wert zum Zeitpunkt V1 und Menopausenalter ein Nomogramm. Alter, AMH-Wert und AFC (V1) korrelierten signifikant mit der Zeitdauer bis zur Menopause ( $\mathrm{p}<0,001)$ und vermochten häufig das Menopausenalter korrekt vorherzusagen (C-Statistik; o,87, o,86 bzw. o,84). Nach der Adjustierung für den Faktor Alter galt dies nur noch für AMH (C-Statistik; 0,90). Als Beispiel angeführt wurde eine 30-jährige Frau mit einem AMH-Wert von $0,15 \mathrm{ng} \bullet \mathrm{ml}^{-1}$, der dem fünften Perzentil der altersspezifischen AMH-Serumkonzentration entsprach. Ihr geschätztes medianes Menopausenalter betrug demnach 48,8 Jahre - das fünfte bis 95. Perzentil entsprach einem Alter von 42,1-53 Jahren. Für eine Frau gleichen Alters mit einem AMHWert von 4,38 ng• $\mathrm{ml}^{-1}$, der dem 95. Perzentil der altersspezifischen AMH-Serumkonzentration entsprach, betrug das geschätzte mediane Menopausenalter dagegen 55,3 Jahre - das fünfte bis 95 . Perzentil entsprach einem Alter von 47,760,1 Jahren. Hieraus wurde die Schlussfolgerung gezogen, dass mit AMH-Wert und Alter die Zeitspanne vorhergesagt werden kann, in der die individuelle Menopause eintreten wird.

\section{Kommentar}

Mit der Frage, zu welchem präzisen Zeitpunkt die Menopause eintreten wird, wer- den die meisten Gynäkologen fast täglich konfrontiert, wenn mit Patientinnen Probleme wie Blutungsstörungen oder die Kontrazeptionsnotwendigkeit in der Perimenopause besprochen werden. AMH gilt als bester Marker der ovariellen Reserve. Bislang ist jedoch nur eine sehr vage Aussage zum erwarteten individuellen Menopausenalter möglich. Somit kann beispielsweise die Frage, ob Kontrazeption notwendig ist, nicht eindeutig beantwortet werden. Diesbezüglich konnte in einer früheren Studie gezeigt werden, dass AMH 5 Jahre vor der Menopause unter die Nachweisgrenze fällt [1].

\section{Die Studienergebnisse haben nur für normoovulatorische Frauen Aussagekraft}

In der vorliegenden Studie wurde die Beziehung zwischen AMH und dem Zeitpunkt der zukünftigen Menopause bei normoovulatorischen Frauen untersucht. Die Ergebnisse haben somit nur für normoovulatorische Frauen Aussagekraft. Das konstruierte Nomogramm hat für die jüngere, ovulatorische Frau, die ihr Menopausenalter abschätzen möchte, einen guten prädiktiven Wert - die prädiktiven Bereiche des Menopausenalters umfassen für alle AMH-Perzentile eine Zeitspanne von 10 Jahren. Keinen prädiktiven Wert besitzt AMH jedoch für Frauen mit unregelmäßigen Zyklen in einem Alter von fast 50 Jahren. Gerade diese Frauen würden aber wahrscheinlich am meisten von

Verantwortlich für diesen Beitrag: Deutsche Menopause Gesellschaft e.V. 
einer exakten Vorhersage des Menopausenalters profitieren. Eine weitere Einschränkung der Studie ist, dass aufgrund der Zusammenführung verschiedener Kohorten auch 2 unterschiedliche Verfahren für die AMH-Bestimmung eingesetzt wurden. Dennoch ist die Studie ein Schritt in die richtige Richtung. Sie untermauert erneut die Bedeutung von AMH als bestem Marker der Eizellreserve.

\section{Korrespondenzadresse}

\section{PD Dr. P. Stute}

Inselspital Bern

Effingerstr. 102, 3010 Bern

Schweiz

petra.stute@insel.ch

Interessenkonflikt. Keine Angabe

\section{Literatur}

1. Sowers MR, Eyvazzadeh $A D, M c C o n n e l l ~ D$ et al (2008) Anti-mullerian hormone and inhibin B in the definition of ovarian aging and the menopause transition. J Clin Endocrinol Metab 93:34783483

\section{Arzneimittel für neuartige Therapien: wissenschaftliche, medizinische, ökonomische und ethische Aspekte}

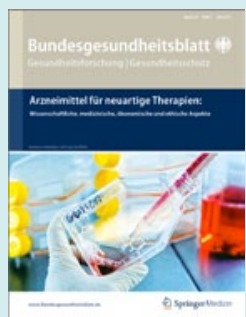

Stammzellforschung und regenerative Medizin stehen in besonderer Weise für den aktuellen und zukünftigen medizinischen Fortschritt. Erfolgreiche

Forschungsinitiativen haben bereits jetzt zur Entwicklung einer beständig wachsenden Zahl von Arzneimitteln für neuartige Therapien geführt, einer sehr heterogenen Gruppe innovativer und teils hoch komplexer Arzneimittel. Die Ausgabe 07/11 der Zeitschrift Bundesgesundheitsblatt mit dem Leitthema „Arzneimittel für neuartige Therapien: wissenschaftliche, medizinische, ökonomische und ethische Aspekte" beschäftigt sich mit der Weiterentwicklung des Feldes unter behördlichen und gesetzlichen Rahmenbedingungen. Neben aktuellen wissenschaftlichen Erkenntnissen und deren Umsetzung in die medizinische Praxis werden auch die ökonomischen Gegebenheiten und ethische Fragen erörtert.

Das Leitthemenheft beinhaltet $u$. a. Beiträge zu folgenden Themen:

- Potenzial hämatopoetischer Stammzellen als Ausgangsmaterial für Arzneimittel für neuartige Therapien

- Klinische Behandlung von Gelenkknorpelschäden mit Hilfe autologer Vorläuferzellen

- Regulatorischer Rahmen für neuartige Therapien

- Die Zertifizierung neuartiger Therapien

- Erfahrungsbericht aus dem Ausschuss für neuartige Therapien (CAT)

- Der lange Weg zur zentralen Zulassung

- Arzneimittel für neuartige Therapien umfassend bewerten

- Erstattungsfähigkeit neuartiger Arzneimitteltherapien
Bestellen Sie diese Ausgabe zum Preis von EUR 13,50 zzgl. Versandkosten bei:

Springer Customer Service Center GmbH Kundenservice Zeitschriften

Haberstr. 7

69126 Heidelberg

Tel.: +49 6221-345-4303

Fax: +49 6221-345-4229

leserservice@springer.com

P.S. Vieles mehr rund um Ihr Fachgebiet finden Sie auf www.springermedizin.de 